\title{
Association between MBL2 haplotypes and dengue severity in children from Rio de Janeiro, Brazil
}

\author{
Alice Maria de Magalhães Ornelas ${ }^{1 *}$, Caroline Xavier-de-Carvalho ${ }^{2}$, Lucia Elena Alvarado-Arnez ${ }^{2 *}$, \\ Marcelo Ribeiro-Alves ${ }^{3}$, Átila Duque Rossi ${ }^{1}$, Amilcar Tanuri ${ }^{1}$, Renato Santana de Aguiar ${ }^{1}$, \\ Milton Ozório Moraes ${ }^{2}$, Cynthia Chester Cardoso ${ }^{1 /+}$
}

'Universidade Federal do Rio de Janeiro, Instituto de Biologia, Departamento de Genética, Laboratório de Virologia Molecular, Rio de Janeiro, RJ, Brasil

${ }^{2}$ Fundação Oswaldo Cruz-Fiocruz, Instituto Oswaldo Cruz, Laboratório de Hanseníase, Rio de Janeiro, RJ, Brasil

${ }^{3}$ Fundação Oswaldo Cruz-Fiocruz, Instituto Nacional de Infectologia Evandro Chagas, Laboratório de Pesquisa Clínica em DST-AIDS, Rio de Janeiro, RJ, Brasil

BACKGROUND Dengue is an arthropod-borne viral disease with a majority of asymptomatic individuals and clinical manifestations varying from mild fever to severe and potentially lethal forms. An increasing number of genetic studies have outlined the association between host genetic variations and dengue severity. Genes associated to viral recognition and entry, as well as those encoding mediators of the immune response against infection are strong candidates for association studies.

OBJECTIVES The aim of this study was to investigate the association between MBL2, CLEC5A, ITGB3 and CCR5 genes and dengue severity in children.

METHODS A matched case-control study was conducted and 19 single nucleotide polymorphisms (SNPs) were investigated.

FINDINGS No associations were observed in single SNP analysis. However, when MBL2 SNPs were combined in haplotypes, the allele rs7095891G/rs1800450C/ rs1800451C/rs4935047A/rs930509G/rs2120131G/rs2099902C was significantly associated to risk of severe dengue under $\alpha=0.05(\mathrm{aOR}=4.02 ; \mathrm{p}=0.02)$. A second haplotype carrying rs $4935047 \mathrm{G}$ and rs7095891G alleles was also associated to risk $(\mathrm{aOR}=1.91 ; \mathrm{p}=0.04)$.

MAIN CONCLUSIONS This is the first study to demonstrate the association between $M B L 2$ haplotypes and dengue severity in Brazilians including adjustment for genetic ancestry. These results reinforce the role of mannose binding lectin in immune response to DENV.

Key words: dengue - polymorphisms - MBL2 - CCR5 - ITCB3 - CLEC5A

Dengue is a neglected tropical disease caused by Dengue virus (DENV), which has four circulating serotypes (DENV-1 to 4 ) and is transmitted through the bite of Aedes mosquitoes. In humans, the majority of infections are asymptomatic or presenting mild flu-like symptoms, known as dengue fever. However, a small proportion of the cases may also present the life-threatening forms such as dengue shock syndrome. ${ }^{(1)}$

Recent estimates suggest an annual occurrence of 390 million infections worldwide.(2) Dengue infection affects infants, young children and adults, and is responsible for 250,000-500,000 hospitalisations and approximately 25,000 deaths each year, mostly among children. (3) In Americas, The Southern Cone subregion reported a total of 254,453 dengue cases in 2017 from which, 99\% were reported in Brazil. ${ }^{(4)}$

doi: 10.1590/0074-02760190004

Financial support: CNPq (404241/2012-3), FAPERJ (E-26/010.001562/2014).

This study was also financed in part by the CAPES (Finance Code 001).

${ }^{*}$ Current address: Universidad Franz Tamayo, Coordinación de Investigación, Cochabamba, Bolivia.

+ Corresponding author: cynthiac@biologia.ufrj.br

(D) https://orcid.org/0000-0003-0565-7047

Received 8 January 2019

Accepted 11 April 2019
Several risk factors influence evolution to severe disease in dengue-infected individuals. Among these factors are secondary infection, known as antibody-dependent enhancement, viral strain differences, viral load, potent host inflammatory immune response, that includes T-cell response, cytokine storm, vascular leakage and complement activation and host genetic background..$^{(1,5)}$ Therefore, disease severity is a complex phenomenon and depends on numerous interactions between human genetic and immunological characteristics as well as viral factors. ${ }^{(1,6,7)}$

The influence of the host genetic background in susceptibility to severe dengue has been evidenced for different populations using either candidate genes or genomewide approaches. ${ }^{(6)}$ A genome-wide association study has reported the association between $M I C B$ and PLCE genes and dengue shock syndrome in children from Vietnam. ${ }^{(8)}$ Moreover, dengue outcomes have already been associated to HLA alleles as well as genes encoding the cell receptor DC-SIGN, CLEC5A, Fc receptors, and molecules such as CTLA-4, TGF- $\beta$ and MBL-2. ${ }^{(6,7)}$ However, conflicting results are still observed and the complete dissection of host genetic factors influencing dengue clinical course still depends on the development of independent studies including a careful control for genetic background.

Mannose-binding lectin (MBL) is a pattern recognition receptor which recognises specific sugar groups on the surface of various microorganisms and triggers 
complement activation to promote pathogen elimination. (9) In dengue infection, this lectin is required for complement-mediated viral neutralisation. ${ }^{(10)} \mathrm{MBL}$ is encoded by $M B L 2$ gene, which comprises four exons. Variations at codons 54 (rs1800450), 57 (rs1800451) and 52 (rs5030737) as well as promoter polymorphisms have been associated to MBL levels and activity. ${ }^{(11,12)}$ However, the association between $M B L 2$ gene and dengue is still controversial and remains to be clearly characterised.

Another common candidate for association studies in dengue is the C-type lectin domain family 5, member $\mathrm{A}$ (CLEC5A) which has been implicated in dengue lethal outcomes. $^{(13)}$ Upon infection, CLEC5A directly interacts with DENV and stimulates the release of proinflammatory cytokines. ${ }^{(13)}$ We have recently demonstrated an association between the polymorphism rs1285933, located at CLEC5A 3'UTR region, and severe dengue in two Brazilian cohorts. ${ }^{(14,15)}$

In addition to the classical candidates, other genes and molecules have also been investigated in dengue infection, including the coreceptor CCR5 and integrins. Recently, $C C R 5$ has been described as a host factor required not only for DENV-2 replication but also for the development of the disease in macrophages. ${ }^{(16)} \beta 3$ integrins are abundant on the surface of vascular endothelial cells and platelets, and play an important role in maintaining vascular integrity, ${ }^{(17)}$ suggesting a possible association to severe dengue. In fact, $\alpha 5 \beta 3$ integrins are commonly used as receptors or coreceptors by several viruses, including DENV. ${ }^{(18)}$

We have previously investigated the role of 11 candidate single nucleotide polymorphisms (SNPs) in four genes including CLEC5A, which was associated to dengue severity, and CCR5. ${ }^{(14)}$ In the present study, we have increased the number of genes/polymorphisms investigated in the same cohort by including new markers at CLEC5A and CCR5, in addition to 13 markers at MBL2 and $I T G B 3$ genes. Proportions of African, European and Native American genetic ancestries were also estimated to adjust for confounding.

\section{MATERIALS AND METHODS}

Subjects and study design - The case group included 87 children with severe dengue admitted in four pediatric intensive care units from Rio de Janeiro - IFF/FIOCRUZ; Instituto de Puericultura e Pediatria Martagão Gesteira, IPPMG/UFRJ; Prontobaby, Hospital da Criança; and Hospital Municipal Jesus - between 2007 and 2008 , aged up to 18 years. Severe dengue was defined in a previous study, according to clinical symptoms as described by Brazilian Ministry of Health and WHO. $(14,19)$ The patients exhibited symptoms consistent with shock syndrome, including slow capillary filling, cold clammy skin, filiform or absent pulse in the presence of either hypotension for age or narrow pulse pressure. Other typical symptoms of dengue were considered such as fever, hemorrhagic manifestations, hemoconcentration, constant vomits, persistent abdominal pain, hypotension, pleural or pericardial effusion or ascites and high levels of AST and ALT. Detailed information about case definition, inclusion/exclusion criteria and ethics statement was described in a previous study. ${ }^{(14)} \mathrm{A}$ control group was selected among neighbors and cases' household members matched by age (up to three years older or younger than the cases). A total of 325 controls were initially included in the study. After serological tests, the rate of dengue IgG seropositivity was $60.6 \%$ and, therefore, the final control group included 197 individuals. Considering a minor allele frequency of $0.05, \alpha=0.05$ and the number of cases and $\mathrm{IgG}+$ controls available, the minimum OR value to achieve $80 \%$ of power was 3.4 .

Individual estimates of African, European, and $\mathrm{Na}-$ tive American genetic ancestries were determined using 28 ancestry informative markers, as described. ${ }^{(20)}$ Distributions of age, gender and genetic ancestry are shown for cases and $\mathrm{IgG}+$ controls in Table I.

Candidate SNPs selection - Candidate SNPs were selected for genotyping based on their classification as a tag to a haplotype or location in coding and/or regulatory regions of genes using HapMap database (www. hapmap.org), Snpper tool (http://snpper.chip.org/) and/ or dbSNP database (http://ncbi. nlm.nih.gov/SNP/). SNPs with minor allele frequencies (MAF) of at least 0.05 at $M B L 2, I T G B 3, C C R 5$ and CLEC5A genes were selected. For $C L E C 5 A$, a $10 \mathrm{~kb}$ upstream region was also analysed for SNP selection to increase coverage. The tag SNPs were selected from HapMap database (phase II Oct 14, on NCBI B36 assembly, dbSNP b126) using data from YRI (Yoruba in Ibadan, Nigeria) and CEU (Utah residents with ancestry from northern and western Europe) populations. A minor allele frequency of 0.01 and $\mathrm{r} 2 \geq 0.8$ was applied as criteria for tag definition. According to these criteria, 22 polymorphisms were selected for analysis [Supplementary data (Table I)], including eight polymorphisms at MBL2 (rs930509, rs4935047, rs2099902, rs2120131, rs1800451, rs1800450, rs5030737 and rs7095891), four SNPs at CLEC5A

TABLE I

Distribution of age, gender and genetic ancestry in dengue severe cases and controls

\begin{tabular}{lcc}
\hline Variable & $\begin{array}{c}\text { Cases } \\
\mathrm{N}=87\end{array}$ & $\begin{array}{c}\text { IgG+ controls } \\
\mathrm{N}=197\end{array}$ \\
\hline Age (years) & $10.03 \pm 3.79$ & $10.02 \pm 3.57$ \\
\hline Gender & & \\
\hline Male & $42(48 \%)$ & $111(44 \%)$ \\
\hline Female & $45(52 \%)$ & $86(56 \%)$ \\
\hline Genetic ancestry (\%) & & \\
\hline European* & $58 \pm 20$ & $50 \pm 24$ \\
\hline \multicolumn{1}{c}{ African } & \\
\hline Native American & $27 \pm 18$ & $35 \pm 22$ \\
\hline
\end{tabular}

Results are represented as mean standard \pm deviation for age and genetic ancestry. ${ }^{*}: \mathrm{p}<0.05$ for comparisons between cases and $\mathrm{IgG}+$ controls. 
(rs1285950, rs1594777, rs1285948 and rs2570407), six at ITGB3 (rs11655943, rs4629025, rs884696, rs7209700, rs3809865 and rs5918) and, finally, four SNPs at CCR5 gene (rs1800940, rs2856762, rs3176763 and rs3087253).

SNP genotyping - Genomic DNA was extracted from peripheral blood by using a salting-out protocol. All SNPs were genotyped using 5' nuclease assays for allelic discrimination by real-time polymerase chain reaction (PCR) using QuantStudio ${ }^{\mathrm{TM}}$ 12K Flex Real-Time PCR System with OpenArray ${ }^{\circledR}$ Block (ThermoFisher Scientific, MA, USA). Reactions were performed in customised arrays according to the manufacturer's instructions. The SNPs rs1285950, rs1594777, and rs1285948 at CLEC5, could not be included in the array due to a failure in probe design.

In silico analysis of MBL2 SNPS - Potential functional impact for $M B L 2$ SNPs was assessed using five different algorithms. Combined annotation dependent depletion (CADD) (v1.4) and deleterious annotation of genetic variants using neural networks (DANN) are evolutionary-based approaches which infer functional impact through data simulation. Functional analysis through hidden markov models (FATHMM-MKL) is an integrative algorithm that uses functional annotations and sequence conservation data through machine learning approach. Finally, PolyPhen 2 and sorting intolerant from tolerant (SIFT) are structure-based approaches that infer functional impact according to sequence conservation and severity of the amino acid change. ${ }^{(21)}$ Functional impact scores were interpreted here following developers' recommendations. In addition, data from The Genotype-Tissue Expression (GTEx) project(22) were also analysed to search for eQTLs using each SNP as query in the GTEx portal.

Statistical analysis - Deviations from Hardy-Weinberg equilibrium (HWE) were assessed by Chi-square test using samples from $\mathrm{IgG}+$ controls $(\mathrm{N}=197)$. Frequencies of each genotype, allele, and minor allele carriers in cases and controls were determined by direct counting. The genotypic, allelic and minor allele carriers association with severe dengue were assessed using odds-ratios (OR) and corresponding 95\% confidence interval (95\%CI) estimated by the fit of logistic generalised linear mixed effect models (L-GLMM) by maximum likelihood, where clusters of neighbors and/or household members were modeled as aleatory effects. Degrees of freedom for fitted effects were approximated by the Satterthwaite method. ${ }^{(23)}$ Gender and African and Native American ancestry estimates were included as fixed effects in multivariate analysis to adjust for confounding (aOR). Linkage disequilibrium (LD) patterns were assessed using the $\mathrm{r} 2$ statistic also using samples from the complete control group. Haplotype L-GLMM analysis was performed with SNPs, not in LD. The allelic phases were estimated by the expectationmaximisation method, and phase uncertainties were treated as sample weights in model fittings. The same strategy was applied to determine the frequency of each haplotype among Africans (AFR), Europeans (EUR), Mixed americans (AMR) and East and South Asians (EAS and SAS) populations from 1000 Genomes Phase 3. ${ }^{(24)}$ Allelic, genotypic, minor allele carriers, and Haplotypic L-GLMM were adjusted for multiple comparisons by false discovery rate (FDR). All analysis was performed in $\mathrm{R}$ version 3.4.4.

Ethics statement - This study was approved by research ethics committee (CAAE3723.0.000.009-08 Instituto Nacional de Infectologia Evandro Chagas/FIOCRUZ). All procedures were performed in accordance with the guidelines of the Helsinki Declaration, as previously described. ${ }^{(14)}$ Informed consent was obtained in two copies from the parents of all subjects enrolled in the study. A signed copy saved with the clinical researcher and the other with the family.

\section{RESULTS}

Quality control and single SNP association analyses - A total of 19 candidate SNPs [Supplementary data (Table I)] was genotyped in 87 cases and 325 controls. SNPs rs5030737 (MBL2) and rs1800940 (CCR5) were excluded from analysis because they were not informative (monomorphic) in our sample. The remaining 17 SNPs were successfully genotyped, with call rates of at least $90 \%$. In all SNPs tested, genotype frequencies were found in HWE. Results of linkage disequilibrium analyses showed moderate LD between SNPs rs2099902 and rs2120131 at MBL2 $\left(\mathrm{r}^{2}=0.68\right)$ and also between $\mathrm{rs} 4629025$ and rs7209700 at ITGB3 $\left(\mathrm{r}^{2}=0.61\right)$. A minimum $\mathrm{r}^{2}$ of 0.8 was used as cutoff for tag SNPs definition. Therefore, all SNPs were included in association analyses.

Frequencies of each SNP were compared between severe cases and IgG+ controls. Results of L-GLMM did not show any statistically significant association when each SNP was analysed independently either before or after adjustment for gender and proportions of African and Native American ancestries [Table II; Supplementary data (Table II)].

Haplotype analyses - When MBL2 SNPs were combined in haplotypes, two combinations carrying allele rs7095891G (haplotypes 2 and 6), in addition to other regulatory variations, were associated to increased risk of severe dengue (Table III). Haplotype 2 (rs7095891G/ $\mathrm{rs} 1800450 \mathrm{C} / \mathrm{rs} 1800451 \mathrm{C} / \mathrm{rs} 4935047 \mathrm{~A} / \mathrm{rs} 930509 \mathrm{G} /$ rs $2120131 \underline{G} / \mathrm{rs} 2099902 \mathrm{C})$ was significantly associated to risk $(\mathrm{aOR}=4.02 ; 95 \% \mathrm{CI}: 1.23-13.09 ; \mathrm{p}=0.02)$ as compared to the reference $(\mathrm{A} / \mathrm{C} / \mathrm{C} / \mathrm{A} / \mathrm{G} / \mathrm{T} / \mathrm{T})$. Haplotype $6(\underline{\mathrm{G}} / \mathrm{C} / \mathrm{C} / \underline{\mathrm{G}} / \mathrm{G} / \mathrm{T} / \mathrm{T})$, with higher frequency, and carrying rs4935047 $\underline{\mathrm{G}}$ in addition to $\mathrm{rs} 7095891 \underline{\mathrm{G}}$ was also associated to risk $(\mathrm{aOR}=1.91 ; 95 \% \mathrm{CI}: 1.02-3.6 ; \mathrm{p}=0.04)$. After FDR adjustment, however, all p-values were above 0.05 , due to a clear loss in statistical power.

Comparisons with data obtained from populations of 1000 Genomes project showed similar frequencies of the high risk haplotype 2 between data from the present study (0.03, including cases and controls) and among Africans (AFR; 0.04), Mixed Americans (AMR; 0.02) and Europeans (EUR; 0.04). The lowest frequencies were observed among East and South Asians (0.003 and 0.007, respectively). For haplotype 6, frequencies varied from 0.12 (AFR) to 0.40 (AMR) [Supplementary data (Table III)]. 
TABLE II

Distribution of $M B L 2$ single nucleotide polymorphisms (SNPs) and analysis of association to dengue severity in children from Rio de Janeiro

\begin{tabular}{|c|c|c|c|c|c|}
\hline SNP & Genotypes & Cases* $^{*}$ & Controls* & OR (95\% CI; p-value) & OR $(95 \% \text { CI; p-value })^{* *}$ \\
\hline \multirow{5}{*}{ rs7095891 } & GG & $42(50.0)$ & $73(39.7)$ & reference & Reference \\
\hline & GA & $36(42.9)$ & $82(44.5)$ & $0.78(0.44-1.41 ; p=0.42)$ & $1.01(0.51-2.00 ; p=0.98)$ \\
\hline & AA & $6(7.1)$ & $29(15.8)$ & $0.44(0.16-1.16 ; p=0.10)$ & $0.42(0.13-1.34 ; p=0.14)$ \\
\hline & Total & 84 & 184 & & \\
\hline & A carriers & $42(50.0)$ & $111(60.3)$ & $0.70(0.40-1.23 ; \mathrm{p}=0.21)$ & $0.85(0.44-1.64 ; p=0.63)$ \\
\hline \multirow{5}{*}{ rs 1800450} & $\mathrm{CC}$ & $65(77.4)$ & $145(81.0)$ & Reference & Reference \\
\hline & $\mathrm{CT}$ & $19(22.6)$ & $31(17.3)$ & $1.43(0.75-2.73 ; p=0.28)$ & $1.47(0.73-2.99 ; p=0.28)$ \\
\hline & TT & $0(0)$ & $3(1.7)$ & n.d & n.d \\
\hline & & 84 & 179 & & \\
\hline & $\mathrm{T}$ carriers & $19(22.6)$ & $34(19.0)$ & $1.31(0.69-2.48 ; p=0.41)$ & $1.32(0.66-2.63 ; p=0.43)$ \\
\hline \multirow{5}{*}{ rs1800451 } & $\mathrm{CC}$ & $68(85.0)$ & $145(83.8)$ & Reference & Reference \\
\hline & $\mathrm{CT}$ & $12(15.0)$ & $27(15.6)$ & $1.26(0.57-2.78 ; p=0.57)$ & $1.51(0.63-3.64 ; p=0.35)$ \\
\hline & TT & $0(0.0)$ & $1(0.6)$ & n.d & n.d \\
\hline & Total & 80 & 173 & & \\
\hline & $\mathrm{T}$ carriers & $12(15.0)$ & $28(16.2)$ & $1.15(0.53-2.53 ; p=0.72)$ & $1.33(0.56-3.13 ; p=0.52)$ \\
\hline \multirow{5}{*}{ rs4935047 } & AA & $21(29.2)$ & $69(40.6)$ & Reference & Reference \\
\hline & AG & $34(47.2)$ & $74(43.5)$ & $1.18(0.57-2.44 ; p=0.65)$ & $1.17(0.53-2.56 ; p=0.70)$ \\
\hline & GG & $17(23.6)$ & $27(15.9)$ & $2.02(0.86-4.75 ; p=0.11)$ & $1.90(0.69-5.24 ; p=0.21)$ \\
\hline & Total & 72 & 170 & & \\
\hline & G carriers & $51(70.8)$ & $101(59.4)$ & $1.42(0.73-2.75 ; p=0.3)$ & $1.34(0.64-2.79 ; p=0.44$ \\
\hline \multirow{5}{*}{ rs930509 } & GG & $55(67.9)$ & $135(75.4)$ & Reference & Reference \\
\hline & GC & $25(30.9)$ & $42(23.5)$ & $1.36(0.68-2.68 ; p=0.38)$ & $1.17(0.55-2.52 ; \mathrm{p}=0.68)$ \\
\hline & $\mathrm{CC}$ & $1(1.2)$ & $2(1.1)$ & n.d & n.d \\
\hline & Total & 81 & 179 & & \\
\hline & $\mathrm{C}$ carriers & $26(32.1)$ & $44(24.6)$ & $1.32(0.67-2.61 ; p=0.43)$ & $1.13(0.52-2.43 ; p=0.76)$ \\
\hline \multirow{5}{*}{ rs2120131 } & TT & $35(45.5)$ & $91(54.2)$ & Reference & Reference \\
\hline & TG & $36(46.7)$ & $62(36.9)$ & $1.79(0.91-3.51 ; \mathrm{p}=0.09)$ & $1.53(0.77-3.05 ; p=0.23)$ \\
\hline & GG & $6(7.8)$ & $15(8.9)$ & $1.43(0.42-4.91 ; p=0.57)$ & $1.20(0.33-4.36 ; p=0.78)$ \\
\hline & Total & 77 & 168 & & \\
\hline & G carriers & $42(54.5)$ & $77(45.8)$ & $1.73(0.90-3.32 ; p=0.10)$ & $1.48(0.76-2.89 ; p=0.25)$ \\
\hline \multirow{5}{*}{ rs2099902 } & TT & $33(42.3)$ & $70(40.2)$ & Reference & Reference \\
\hline & $\mathrm{TC}$ & $34(43.6)$ & $77(44.3)$ & $1.01(0.54-1.89 ; p=0.97)$ & $1.28(0.65-2.56 ; p=0.47)$ \\
\hline & $\mathrm{CC}$ & $11(14.1)$ & $27(15.5)$ & $0.97(0.37-2.49 ; p=0.94)$ & $1.15(0.41-3.25 ; p=0.79)$ \\
\hline & Total & 78 & 174 & & \\
\hline & C carriers & $45(57.7)$ & $104(59.8)$ & $1.04(0.44-2.49 ; \mathrm{p}=0.92)$ & $1.00(0.39-2.61 ; p=0.99)$ \\
\hline
\end{tabular}

*: results are shown as $\mathrm{N}(\%)$ for cases and $\mathrm{IgG}+$ controls. **: odds-ratios $(\mathrm{OR})$ and $\mathrm{p}$-value adjusted for gender and $\mathrm{African}$ and Native American genetic ancestries; n.d = not done.

Considering the moderate LD between SNPs rs2099902 and rs2120131 $\left(r^{2}=0.68\right)$, haplotype analyses were also performed removing one of these markers (rs2099902). Results obtained showed that the haplotype rs7095891G/ rs1800450C/ rs1800451C/ rs4935047G/
rs930509G/ rs2120131T was associated to risk, with OR values similar to those obtained in the complete haplotype $6(\mathrm{aOR}=1.84 ; 95 \% \mathrm{CI}$ : 0.99-3.44; $\mathrm{p}=0.055)$, using $\mathrm{A} / \mathrm{C} / \mathrm{C} / \mathrm{A} / \mathrm{G} / \mathrm{T}$ as reference. On the other hand, the association between the low frequency haplotype $\underline{\mathrm{G}} / \mathrm{C} / \mathrm{C} / \mathrm{A} /$ 
$\mathrm{G} / \underline{\mathrm{G}}$ including rs7095891 $\underline{\mathrm{G}}$ and rs2120131G alleles, was softened after removing $\mathrm{rs} 2099902(\mathrm{aOR}=2.69 v s \mathrm{4.02})$, with a consequent reduction in power $(\mathrm{p}=0.1)$. SNPs at ITGB3 and CCR 5 genes were also combined in haplotypes, but no association was found (data not shown).

In silico prediction of MBL2 SNPs functional effect - Possible functional impacts of $M B L 2$ variations were investigated in silico. Results obtained from five different algorithms have classified the nonsynonymous polymorphisms rs1800450, rs1800451 and rs5030737 as functional or potentially pathogenic (Table IV). Moreover, when the intron variations rs 930509 and rs 4935047 were queried at GTEx browser, allele $\mathrm{G}$ at both sites was associated to higher MBL2 expression in liver ( $\mathrm{p}=7.2$ $\mathrm{e}^{-13}$ for rs930509 and $\mathrm{p}=1.3 \mathrm{e}^{-4}$ for rs4935047).

\section{DISCUSSION}

Dengue susceptibility is a complex phenotype that depends on components intrinsic to the virus and the host. Genetic heterogeneity of molecules involved in the host immune response to DENV infection has been supported to play a key role in the development of severe disease ${ }^{(6)}$ Here, we investigated the association between severe dengue and 19 candidate SNPs in genes encoding critical molecules involved in the host immunological resistance against DENV infection, including mannosebinding lectin, CLEC5A, $\beta 3$ integrin, and CCR5.

Mannose-binding lectin acts in first line response to DENV ${ }^{(6,10)}$ A study of Vietnamese subjects has detected no association between the variation Gly54Asp (rs1800451) and dengue clinical outcomes. ${ }^{(25)}$ By contrast, studies from Northeast Brazilian groups have both reported associations between combinations of functional MBL2 exon 1 SNPs at codons 52 (rs5030737), 54 (rs1800450) and 57 (rs1800451) and dengue outcomes such as thrombocytopenia ${ }^{(26)}$ and also dengue haemorrhagic fever (DHF). ${ }^{(27)}$ Here, we have detected an association between two MBL2 haplotypes and increased risk of severe dengue. The strongest association was observed for haplotype 2 , which differed from the reference haplotype by carrying the alleles rs $7095891 \mathrm{G}, \mathrm{rs} 2099902 \mathrm{C}$ and $\mathrm{rs} 2120131 \mathrm{G}(\mathrm{aOR}=4.02 ; \mathrm{p}$ $=0.02$ ). As expected, similar frequencies of this haplotype were obtained among AFR, AMR and EUR populations from 1000 Genomes project $(0.04,0.02$ and 0.04 , respectively). The lowest frequencies were observed among East (0.003) and South Asians (0.007) [Supplementary data (Table III)], suggesting that large sample sizes would be required to replicate our findings in populations with this genetic background.

The SNP rs7095891 is located at MBL2 5'UTR, while rs2099902 and rs2120131 are located at 3'UTR region. In silico predictions have suggested that $3^{\prime}$ UTR SNPs such as rs2099902 and rs2120131, could affect miRNA binding site, while intronic and $5^{\prime}$ near gene SNPs as rs7095891 could affect transcription binding sites. ${ }^{(28)}$ In fact, data obtained from the GTEx project have suggested that the intron variations rs930509 and rs4935047 act as eQTLs for MBL2 gene in liver. However, data from other tissues and cell types were not available for analysis. The allele rs $7095891 \mathrm{G}$ also referred as $M B L 2^{*} Q$, has also

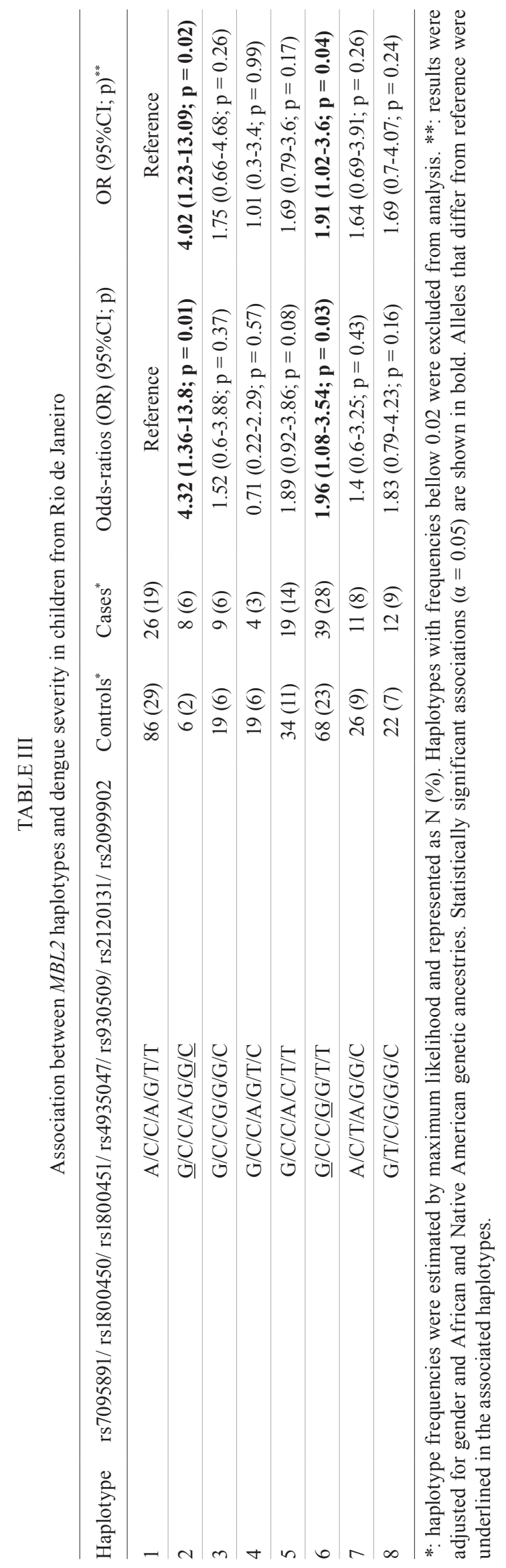


TABLE IV

In silico prediction of functional effects of $M B L 2$ single nucleotide polymorphisms (SNPs) according to five algorithms

\begin{tabular}{lcccccc}
\hline SNP ID & $\begin{array}{c}\text { Gene region } \\
\text { (function) }\end{array}$ & $\begin{array}{c}\text { CADD } \\
\text { (PhRed score) }\end{array}$ & $\begin{array}{c}\text { DANN } \\
\text { score }\end{array}$ & $\begin{array}{c}\text { FATHMM } \\
\text { (MKL score) }\end{array}$ & PolyPhen-2 & SIFT \\
\hline rs2099902 & 3'UTR & 0.520 & n.d & 0.00014 & n.d & n.d \\
\hline rs2120131 & 3'UTR & 1.190 & n.d & 0.03244 & n.d & n.d \\
\hline rs930509 & Intron & 3.006 & n.d & 0.06633 & n.d & n.d \\
\hline rs4935047 & Intron & 6.217 & n.d & 0.02010 & n.d & n.d \\
\hline rs1800451 & Exon & 24.6 & 0.997 & 0.675 & Damaging & 0.001 \\
\hline rs1800450 & Exon & $\mathbf{2 5 . 5}$ & $\mathbf{0 . 9 9 9}$ & $\mathbf{0 . 6 9 1}$ & Damaging & $\mathbf{0 . 0 3}$ \\
\hline rs5030737 & Exon & $\mathbf{2 5 . 8}$ & $\mathbf{0 . 9 9 9}$ & 0.091 & Damaging & $\mathbf{0 . 0 0 1}$ \\
\hline rs7095891 & Upstream & 0.094 & n.d & 0.00396 & n.d & n.d
\end{tabular}

Scores higher than 20 (combined annotation dependent depletion - CADD), 0.91 (deleterious annotation of genetic variants using neural networks — DANN), 0.5 (functional analysis through hidden markov models — FATHMM-MKL) and lower than 0.05 (sorting intolerant from rolerant — SIFT), were considered pathogenic; n.d: not done.

been associated to variations in $M B L 2$ serum levels depending on combinations with promoter SNPs. ${ }^{(11,12)}$ Taken together, the literature data suggest that $M B L$ levels may be regulated by variations along all gene sequence.

The OR values towards risk suggest that haplotypes 2 and 6 might be associated to lower $M B L$ levels, since deficiency of this receptor have been associated to severe dengue phenotypes. ${ }^{(27,29)}$ However, further analyses are still required to determine the correlation between the associated haplotypes and MBL-2 levels during DENV infection. A possible interaction between genetic variations and classical risk factors such as secondary infection also remains to be determined.

The variations at ITGB3,CLEC5A and CCR 5 genes were not associated to severe dengue in our cohort either independently or combined in haplotypes. The protein encoded by ITGB3 gene ( $\beta 3$ integrin) is abundant in surface receptors of vascular endothelial cells and platelets and has been described as a receptor for DENV in these cells. ${ }^{(18)}$ CCR5 plays a role in DENV-2 replication and disease development in human and mouse macrophages. (16) The CCR5 432 has been previously investigated in our cohort $^{(14)}$ as well as in a cohort from Australia and, accordingly, no association was found. ${ }^{(30)}$

CLEC5A is critical for dengue-virus-induced lethal disease. ${ }^{(13)}$ Our group has previously shown an association between a SNP at CLEC5A 3' near-gene (rs1285933) and severe dengue using the same individuals investigated in this study. ${ }^{(14)}$ The association was replicated later in patients from another Brazilian region. ${ }^{(15)}$ In the current study, we have increased gene coverage by including additional SNPs as a way to better understand the influence of CLEC5A genetic structure in dengue severity. Since no associations were found, the data available to date suggest that the 3' near gene variation rs1285933 may be the functional variant of this gene.

Results presented here reinforce the association between MBL2 gene and dengue severity among Brazilians. Our data also highlight the importance of the haplotype context and linkage disequilibrium patterns, since different $M B L 2$ polymorphisms influence protein expression and activity. Taken together, these results and literature data suggest that $M B L 2$ levels/activity may influence dengue prognosis.

\section{AUTHORS' CONTRIBUTION}

AMMO - Experimental procedures, manuscript preparation; CXC and LEAA - data collection, experimental procedures; ADR - in silico analyses; MRA - statistical analyses; AT and RSA - study design, financial support; MOM and CCC - study design, data analysis, manuscript preparation.

\section{REFERENCES}

1. Guzman MG, Harris E. Dengue. Lancet. 2015; 385(9966): 453-65.

2. Bhatt S, Gething PW, Brady OJ, Messina JP, Farlow AW, Moyes $\mathrm{CL}$, et al. The global distribution and burden of dengue. Nature. 2013; 496(7446): 504-7.

3. Guzman MG, Halstead SB, Artsob H, Buchy P, Farrar J, Nathan MB, et al. Dengue : a continuing global threat Europe PMC Funders Author Manuscripts. Nat Rev Microbiol. 2010; 8(120): S7-16.

4. Salles TS, Sá-Guimarães TE, Seam E, De Alvarenga L, Guimarãesribeiro V, Damião M, et al. History, epidemiology and diagnostics of dengue in the American and Brazilian contexts: a review. Parasit Vectors. 2018; 11: 1-12.

5. Halstead SB. Dengue. Lancet. 2007; 370: 1644-52.

6. Xavier-Carvalho C, Cardoso CC, Kehdy FS, Pacheco AG, Moraes MO. Host genetics and dengue fever. Infect Genet Evol. 2017; 56: 99-110.

7. Wilder-Smith A, Ooi EE, Horstick O, Wills B. Dengue. Lancet. 2019; 393(10169): 350-63.

8. Khor CC, Chau TNB, Pang J, Davila S, Long HT, Ong RTH, et al. Genome-wide association study identifies susceptibility loci for dengue shock syndrome at MICB and PLCE1. Nat Genet. 2011; 43(11): 1139-41.

9. Ip WK, Takahashi K, Ezekowitz Ra, Stuart LM. Mannose-binding lectin and innate immunity. Immunol Rev. 2009; 230(1): 9-21.

10. Avirutnan P, Hauhart RE, Marovich MA, Garred P, Atkinson JP, Diamond MS. Complement-mediated neutralization of dengue virus requires mannose-binding lectin. MBio. 2011; 2(6): e00276-11. 
11. Garred P, Larsen F, Seyfarth J, Fujita R, Madsen HO. Mannose-binding lectin and its genetic variants. Genes Immun. 2006; 7(2): 85-94.

12. Adamek M, Heyder J, Heinold A, Fiedler G, Opelz G, Tran TH. Characterization of mannose-binding lectin (MBL) variants by allele-specific sequencing of MBL2 and determination of serum MBL protein levels. Tissue Antigens. 2013; 82(6): 410-15.

13. Chen ST, Lin YL, Huang MT, Wu MF, Cheng SC, Lei HY, et al. CLEC5A is critical for dengue-virus-induced lethal disease. Nature. 2008; 453(7195): 672-6.

14. Xavier-Carvalho C, Gibson G, Brasil P, Ferreira RX, Santos RS, Cruz OG, et al. Single nucleotide polymorphisms in candidate genes and dengue severity in children: a case-control, functional and meta-analysis study. Infect Genet Evol. 2013; 20: 197-205.

15. Xavier-Carvalho C, Cezar RS, Freire NM, de Vasconcelos CMM, Solorzano VEF, de Toledo-Pinto TG, et al. Association of rs1285933 single nucleotide polymorphism in CLEC5A gene with dengue severity and its functional effects. Hum Immunol. 2017; 78(10): 649-56.

16. Marques RE, Guabiraba R, Del Sarto JL, Rocha RF, Queiroz AL, Cisalpino D, et al. Dengue virus requires the CC-chemokine receptor CCR 5 for replication and infection development. Immunology. 2015 ; 145(4): 583-96.

17. Lauffenburger DA, Horwitz AF. Cell migration: a physically integrated molecular process. Cell. 1996; 84(3): 359-69.

18. Zhang J, Wang J, Gao N, Chen Z, Tian Y, An J. Up-regulated expression of $\beta 3$ integrin induced by dengue virus serotype 2 infection associated with virus entry into human dermal microvascular endothelial cells. Biochem Biophys Res Commun. 2007; 356(3): 763-8.

19. WHO - World Health Organization. Dengue guidelines for diagnosis, treatment, prevention and control: new edition. World Health Organization. 2009. Available from: http://www.who.int/ iris/handle/10665/44188

20. Almeida TB, Costa M, Mendes V, Franciso J, Rafael F, Ferry DA, et al. Drug metabolism and transport gene polymorphisms and efavirenz adverse effects in Brazilian HIV-positive individuals. J Antimicrob Chemother. 2018; 73(9): 2460-7.

21. Lee PH, Lee C, Li X, Wee B, Dwivedi T, Daly M. Principles and methods of in-silico prioritization of non-coding regulatory variants. Hum Genet. 2018; 137(1): 15-30.

22. GTEx Consortium, Aguet F, Brown AA, Castel SE, Davis JR, He $\mathrm{Y}$, et al. Genetic effects on gene expression across human tissues. Nature. 2017; 550(7675): 204-13.

23. Satterthwaite FE. An approximate distribution of estimates of variance components. Biometrics Bull. 1946; 2(6): 110-4.

24. The 1000 Genomes Project Consortium. A global reference for human genetic variation. Nature. 2015; 526: 68-74.

25. Loke H, Bethell D, Phuong CXT, Day N, White N, Farrar J, et al. Susceptibility to dengue hemorrhagic fever in Vietnam: evidence of an association with variation in the vitamin D receptor and $\mathrm{FC} \gamma$ receptor IIA genes. Am J Trop Med Hyg. 2002; 67(1): 102-6.

26. Acioli-Santos B, Segat L, Dhalia R, Brito CAA, Braga-Neto UM, Marques ETA, et al. MBL2 gene polymorphisms protect against development of thrombocytopenia associated with severe dengue phenotype. Hum Immunol. 2008; 69(2): 122-8.

27. Figueiredo GG, Cezar RD, Freire NM, Teixeira VG, Baptista P, Cordeiro M, et al. Mannose-binding lectin gene (MBL2) polymorphisms related to the mannose-binding lectin low levels are associated to dengue disease severity. Hum Immunol. 2016; 77(7): 571-5.

28. Kalia N, Sharma A, Kaur M, Kamboj SS, Singh J. A comprehensive in silico analysis of non-synonymous and regulatory SNPs of human MBL2 gene. Springerplus. 2016; 5(1): 811

29. Alagarasu K, Bachal RV, Bhagat AB, Shah PS, Dayaraj C. Elevated levels of vitamin $\mathrm{D}$ and deficiency of mannose binding lectin in dengue hemorrhagic fever. Virol J. 2012; 9(1): 86.

30. Brestovac B, Halicki LA, Harris RP, Sampson I, Speers DJ, Mamotte $\mathrm{C}$, et al. Primary acute dengue and the deletion in chemokine receptor 5 (CCR5 $\Delta 32$ ). Microbes Infect. 2014; 16(6): 518-21. 